\title{
Extraction of Agar and Alginate from Marine Seaweeds in Red Sea Region
}

\author{
Adiam Abraham ${ }^{1}$, Berihu Afewerki ${ }^{2}$, Biniam Tsegay ${ }^{3}$, Henok Ghebremedhin ${ }^{4}$, Berhane Teklehaimanot $^{5}$ \\ and Kunduru Surender Reddy ${ }^{6^{*}}$ \\ 1,2,3,4,5 Massawa College of Marine Sciences and Technology, Department of Marine Biology, Massawa, Eritrea \\ ${ }^{6}$ Department of Biology, Eritrea Institute of Technology, Mai Nefhi, Asmara, Eritrea
}

Received: July 11, 2018; Accepted: July 29, 2018; Published: August 23, 2018

*Corresponding author: Kunduru Surender Reddy, Department of Biology, Eritrea Institute of Technology, Mai Nefhi, Asmara, Eritrea, E-mail: ksreddy.eit@gmail.com

\begin{abstract}
Water constitutes major portion of the earth's crust approximately (75\%), and it applies to all biological organisms, including sea weeds. Its extraction is useful to human beings directly and indirectly. The study was conducted in Red Sea area of Massawa beaches and extracted agar and alginate from two varieties of red algae (Gracilaria arcuate) and brown algae (Turbinaria decurrens). These extractnats were having many applications for human benefits and also industrial applications such as binding agent and agro-based industry. These were analysed for their phycocolloids extraction (specifically agar and alginate). $20.4 \%$ of agar was extracted from Gracilaria arcuata according to the procedure of Geratrudes (1990). Gracilaria arcuata were pre-treated with $\mathrm{Na}_{2} \mathrm{CO}_{3}$ followed by $\mathrm{NaOH}$ treatment and finally by extraction. The results were found to be encouraging in its powdered product has been obtained. Parallel to that $25.6 \%$ powdered Sodium alginate was extracted by Green process from the extraordinary species of brown algae, Turbinaria decurrens and as per chemical analysis, positive result was obtained. According to the data from Hernandez-Carmona, et al. [9], agar is the most expensive colloid in worlds market, at US $\$ 18$ per $\mathrm{kg}$, followed by alginates and carrageenan at US\$12 and US\$10.5 per $\mathrm{kg}$, respectively. Hence, new methods for extraction are required to obtain high quality byproducts from sea weeds.
\end{abstract}

Keywords: Gracilaria arcuata; Turbinaria decurren; agar; alginic acid; sodium alginate; phycocolloids;

\section{Introduction}

The growing interest in the development of the seaweed resource is primarily due to the recognition of its economic importance as a source of food, as raw material for the manufacture of commercial products such as agar, alginates and carrageenan and its use as an additional source of livelihood for communities that inhabit the coastal areas. Eritrea is having shore line $(1200 \mathrm{~km})$ of the Red Sea and the biodiversity of Red sea is surplus, the exploitation is at its infancy of this region especially the seaweeds and is one of the major untapped resources of Red Sea. Seaweeds or marine macro algae are primitive non-flowering plants without true root, stem and leaves. They form one of the commercially important marine living renewable resources. They are the enormous source for the production of phytochemicals such as agar, carrageenan and alginate. Seaweeds are also called Medical food of the 21st century [18]. Records of seaweeds from localities in Eritrea as well as localities from other coasts of the western Indian Ocean have revealed that the regional distribution of seaweeds in the Red Sea. For the present study [2], it was recorded Gracilaria arcuata and Turbinaria decurrens and was extracted its byproducts alginate and others in the form of insoluble mixed salts of mainly calcium, with lesser amounts of magnesium, sodium, and potassium and is concentrated in the intracellular space [21] and non-nitrogenous extracts from red algae [4].

Standard methods were used to extract. As the first phycocolloid used, agar was one of the first 476 functional ingredients from algae for foods and nutraceutical, even as food ingredients it approved as GRAS (Generally Recognized as Safe) by the FDA (Food and Drug Administration) [16].

\section{Research Objectives}

- To extract agar and alginate from marine seaweeds by using modern techniques

- Application of extractnats

\section{Review of Literature}

\section{Seaweeds in Eritrea}

survey of seaweeds in Eritrea, the taxonomy of specific and infraspecific macro algae seaweeds is as listed below in table 1 and divided according to their amount [2], in which according to the report some islands of the Dah-lak Archipelago and the surroundings of the port town of Massawa includes newly found species of Chlorophyta 26, Phaeophyta 20, and Rhodophyta 55, in 2005. In which generally Rhodophyta species are the most abundant ones in Red sea. 
Table 1: Number of specific and infraspecific taxa of macro algae reported from Eritrea

\begin{tabular}{|c|c|c|c|c|}
\hline Taxonomy & Chlorophyta & Phaeophyta & Rhodophyta & Total \\
\hline Previous Present records & 50 & 108 & 128 & 286 \\
\hline Present report & 26 & 20 & 55 & 101 \\
\hline New records there in & 11 & 7 & 18 & 36 \\
\hline
\end{tabular}

As shown in table 1, species diversity as shown and the algal genera Caloglossa and Chlorodesmis and the species Caulerpella ambigua, Caulerpa fastigiata and C. selago are restricted to the southern part of the Red Sea. Many Red Sea seaweed species have a cosmopolitan distribution. Some taxa are reported to be endemic to the Red Sea, whereas others are found in particular localities in the Red Sea and also in the Indo-West Pacific.

\section{Types of seaweed hydrocolloid}

Seaweeds they do contain polysaccharides which are present inside the cell wall and agar, alginate, and other components.

- Agar: is the general name for polysaccharides, which are dried amorphous gelatin like non-nitrogenous extract from some red algae. Different Seaweeds such as Gelidium, Gracilaria, Pterocladia and Gelidiella are the main sources used for the extraction of agar [8].

- Alginate: is a polysaccharide which occurs in all brown seaweeds as a structural component of the cell wall in the form of insoluble mixed salts of mainly calcium, with lesser amounts of magnesium, sodium, and potassium and is concentrated in the intracellular space. The most important sources are Macrocystis, Ascophyllum, Turbinaria and Sargassum [21].

\section{Sources of Agar}

Different genus of red algae is used as the raw material in agar production as Gelidium agar, Gracilaria agar, Pterocladia agar, for the current research. To describe the product more accurately, it is usual to mention the origin of the seaweeds. Originally Gelidium agar constituted what we consider genuine agar, assigning the term agaroids to the products extracted from other seaweeds.

\section{Agar Extraction and its applications}

The steps required to obtain agar from marine seaweeds are: After collection of seaweeds from the research site it was processed in the following process like cleaning, chemical pretreatment, extraction, filtration, bleaching and dewatering. Other methods are followed like pretreatment, extraction, primary dewatering, and purification by freeze thaw cycle, bleaching and sun-drying. Finally, the processed homogeneous product will be obtained. Slight precautions are required for Gracilaria requires slightly less severe conditions of processing than Gelidium or Gelidiella. Furthermore, the quality of the final product is highly dependent on the quality of the raw material, which, in turn, is dependent on a number of factors:

- Intrinsic (e.g. species of seaweed),
- Environmental (e.g. temperature and salinity of water during growth of seaweed),

- Harvesting (e.g. degree of mixing with other weeds), and

- Post-harvest (e.g. conditions of storage of seaweed prior to processing).

The alternative process relies on syneresis. This is the term used to describe the separation of liquid from a gel. With no refrigeration required, the energy consumption is obviously much lower than for the freeze-thaw method, and, since more water has been removed, less soluble matter remains, so the agar is purer. Less energy is also needed in the drying process since less water is being removed. This process based on syneresis has been widely adopted by large agar producers who can afford the higher capital costs for this equipment.

In extraction process the conquered method of checking the quality of agar produced is through measuring gel strength.

\section{Gel strength}

International trade in dried Gracilaria is based largely on gel strength; the higher the gel strength, the higher the price paid. Its measurement raised some questions: although the processing industry recognizes a standard method, it is not always used by investigators and poor post-harvest handling could play a role.

\section{Food grade agar}

About $90 \%$ of the extracted agar has found extensive applications in food products such as in baked goods industry it's used as a stabilizer and thickener, in meat and fish product as a gelling agent, in dairy products like cream cheese and yoghurt as texture improver. The remaining $10 \%$ is applied for bacteriological medium, gel electrophoresis, chromatography, immunology, and immobilization of enzymes [1]. In electrophoresis agarose gel is widely used as inert supports for the electrophoretic separation and analyze of nucleic acid samples, an essential part of the DNA sequencing process $[15,19]$. In medical fields, agar is also used as a laxative and for the treatment of constipation. Its action in the intestinal tract is comparable to that of cellulose of vegetable foods in aiding bowel movement. Additionally, it is applicable in making dental casts in dentistry, and as a surgical lubricant. The capacity of agar to block streptococcal adhesion to bio surface has made it a potential component in mouthwashes and spray wash for foods [21].

\section{Microbiological agar}

It is specially purified to ensure that it does not contain 
anything that might modify bacterial growth. It is therefore more expensive; frequently at least twice the price of food grade agar. The agar gel should be as clear as possible so that any bacterial growth can be easily seen. This can only be made from species of Gelidium and Pterocladia because the resulting agar has a low gelling temperature $\left(34-36^{\circ} \mathrm{C}\right)$ that allows the addition of other materials to the agar with a minimum risk of damage. Gracilaria and Gelidiella give agars that gel at $41^{\circ} \mathrm{C}$ or higher. "Bacto" agars must not contain anything that might inhibit the growth of bacteria, such as trace metals, soluble carbohydrates or proteins, nor should they contain any bacterial spores. The gels must be strong and have good clarity or transparency. Manufacturers of bacteriological agar keep all processing details confidential [8].

The use of agar in bacteriology is one of the most important uses and requires strict physical-chemical control as well as the absence of hemolytic substances and what is more important and difficult, the absence of any bacterial inhibitors. Robert Koch started using agar in 1881 to gel culture broths when preparing solid culture media and this was the first introduction of this oriental product to Europe.

As agar is used only as a gelling agent in solid media, it is essential to avoid interactions with the rest of the media components such as meat extract, peptones, proteins, amino acids, sugars and other carbohydrates, as well as pigments, indicators, inhibitors, mineral salts, etc., used in their formulation. It has to mix with these components without producing problems such as colour changes, precipitate formation or gel strength losses, even after autoclave sterilization. The rest of the parameters vary as the agars are adapted to the individual requirements of the manufacturer and end user [1]. Gelidium cartilagenium collected along the West Coast of North America is the common source of bacteriological agar in the U.S.A.

\section{Alginate}

“The term 'algin' or 'alginates' is used as a generic name for the salts of alginic acid such as sodium, potassium, ammonium, calcium and Propylene Glycol Alginates (PGAs) and few properties were known at that time. They are quite abundant in nature since they occur both as a structural component in marine brown algae (Phaeophyceae), comprising up to $40 \%$ of the dry matter, and as capsular polysaccharides in soil bacteria and used for the production by microbial fermentation and also by post-polymerization modification of the alginate molecule. All commercial alginates are at present still extracted from algal sources. Algin occurs in the cell wall of all brown algae as a structural component of the cell wall in the form of insoluble mixed salts of mainly calcium, with lesser amount of magnesium, sodium and potassium and is concentrated in the intracellular space [21].

\section{Source of Alginate}

Brown sea weeds do contain alginate in their cell wall but the amount of yield varies among the species like Turbinaria,
Ascophyllum, Durvillaea, Ecklonia, Laminaria, Lessonia, Macrocystis and Sargassum. Although from all Sargassum, is only used.

\section{Extraction and Application of Alginate}

The alginate content in brown sea weed varies depending up on the species, environmental conditions, season of harvest, and method of extraction. Alginates are natural polysaccharides that are extracted from brown seaweeds and widely used for their rheological properties [17].

The alginate industry extraction protocol is divided in to five steps; acidification, alkaline extraction, solid-liquid separation, precipitation and drying. Alkaline extraction is the main step as it corresponds to the extraction phase itself acidified seaweed are immersed in sodium carbonate solution: insoluble alginic acid is converted in to soluble sodium alginate, which passes in to aqueous phase. This step, is conducted in batch reactor, requires high quantities of reactant and water, and several hours are required to attain the optimum production yield, depend in on the seaweed species concerned [17].

There are different extractions protocols of alginate. Some of the common methods are:

Green process: the algae are first pre-extracted with hydrochloric acid then the alginic acid is extracted with excess calcium carbonate the solution [17].

LeGloachec Herters process: in which the algae are first extracted with excess of sodium carbonate, make them not dissolved by floatation, and the solution is precipitated with acid, where by alginic acid is precipitated out.

Batch process: in which it is time, water and reactant consuming [17].

Reactive extrusion: which save time in the processing session

Suzuki method: which specially measure the gel strength?

Alginate and used in the manufacture of soft capsules and is consumed as a beverage of lowering blood sugar level. In the textile industry, it was used as an additive for textile dye.

In the wide range of industrial application, alginates are essential components as thickening, gelling or stabilizing agents, and in some cases, immune stimulatory agents. Alginate has similar applications as agar but tend to be more widely used due to their broader gelling properties. In food, they may be found in products ranging from sauces and jellies to meats, pastry and ice creams. Alginates are also used in the manufacture of diary, bakery, meat, and other products, by applying the gelling, thickening and stabilizing property. Alginate products use as stabilizing agent to give a smooth texture to frozen desserts, and ice cream substitutes, chocolate milk suspensions and various drinks. Propylene Glycol Alginate (PGA) due to its ability to dissolve in the low $\mathrm{pH}$ it is used as emulsifying and thickening 
agent in the products with the low $\mathrm{pH}$ such as sauces, syrups and sherbets. And in field of Aquaculture alginate uses as a binding for animal feeds.

Alginate gels have also a great role in preventing of moisture losses in certain fishes like silver salmon and rock fish. Some studies tell that including the alginate in the diet 1-3\% can reduce Hypertension. In addition, it can also lower the blood glucose level. And in case of bioremediation alginates play a great role by the absorption of the heavy metals. The bio-adsorption capacity of these algae for heavy metals resides mainly in a group of linear polysaccharides known as alginates that occur as a gel in the algal thallus.

Upcoming biotechnological applications, on the other hand, are based either on specific biological effects of the alginate molecule itself or on its unique, gentle, and almost temperatureindependent sol/gel transition in the presence of multivalent cations (e.g., Ca2_), which makes alginate highly suitable as an immobilization matrix for living cells. Alginate extracted from the macroalgae Sargassum sinicola was used as the raw material for co-immobilization of the microalgae Chlorella sorokiniana and growth-promoting bacterium Azospirillum brasilense for waste water treatment and as an inoculants carrier of $A$. brasilense for plant growth promotion.

The industrial applications of alginates are linked to its ability to retain water, and its gelling, viscosifying, and stabilizing properties. Alginates are used extensively in the textile and paper industries. For the industrial uses it acts as the plasticizer of the cement. Also alginates reduce the water penetration and improves mixing and suspension of pigments in clays. Like as a thickening agent in the paints, as an emulsifying agent for vegetable and mineral oils shampoo foam stabilizers. In medicine alginate is used as a wound healing, to stimulate the immune system, and offers in the weight reduction, and offers a potential for weight reduction and also reduction of glycemic index through reduced intestinal absorption and also increases satiety. Alginate is one the materials most employed in practice to make dental impressions. Alginate fiber preparations have been used for some time to suppress bleeding associated with surgical procedures, as wound dressing, and as swabs for culturing.

Sodium alginate is a useful adjuvant in immunization agent to strain of influenza virus. Sodium alginate is also found effective in diminishing in hyper-calciuria in urolithiasis and found useful in the treatment of esophagitis. Alginate immobilized cell systems are used as biocatalysts in several industrial processes such as ethanol production by yeast cell, production of monoclonal antibodies from hybridoma cells [10].

\section{Materials and Methods}

\section{Extraction of agar}

The procedure of Gertrudes (1990) was used with little modification. In agar extraction procedure Gracilaria arcuata were collected 10 times from the coastal reefs of Red Sea near Gurgussum and Hirgigo at low tide session (mostly 6:00 am12:00 pm). The collected samples were cleaned thoroughly with fresh water and sun dried. Then extraction process was followed; there were two phases of extraction, the first phase was preextraction treatment and the second one was extraction phase.

\section{Pre extraction phase}

The pre-extraction treatment involves bleaching and alkali treatment in the processing of Gracilaria samples.

\section{Bleaching}

The dried sample was soaked in tap water and was spread on the table, allowed to dry and then wet again by sprinkling water. The process was repeated until the Gracilaria sample is completely bleached and the bleached seaweed remained brittle and dry.

\section{Bleaching with lime}

A lime $\left(\mathrm{CaCO}_{3}\right)$ solution of $10 \%$ was prepared and sprinkled over the dried Gracilaria samples.

\section{Alkali treatment}

Gracilaria arcuata were treated with aqueous sodium hydroxide solution. The sample was treated with $10 \%$ of sodium hydroxide aqueous solution at $95^{\circ}$ for 90 minutes in water bath. The treated seaweed was washed with tap water until the alkali was almost free. The treated seaweed was again dried by spreading on the table under the fan.

\section{Extraction phase}

Total $100 \mathrm{gm}$ of treated and dried sample was soaked in two beakers with $1000 \mathrm{ml}$ of water in each. They were left to equally distribute to the beakers from the result of pre-extraction treatment process and enough amount of acetic acid was added. After soaking the seaweed samples in the acidic water for 30 minutes, the water was drained off and $800 \mathrm{ml}$ of fresh water was added to each beaker. Then the sample was heated in water bath for one hour at $95^{\circ} \mathrm{C}$. Then the extract was heated for 3 hours and filtered with normal filter instead of filter aids. Resulted extract was putted into pan, and $100 \mathrm{ml}$ of hot water was added into it. The agar solution was allowed to gel at room temperature. Then the gel cut into small squares with a thin blade spatula and the pan was putted in a freezer. The pan was removed from the freezer the next day and was allowed the gel to thaw. Then drained the thawed water, and the gel was washed with tap water till it became colorless. Then the gel was squeezed off and finally treated with isopropanol solution. The isopropanol squeezed off. At last the flaky agar was Shredded, and then putted in a tarred aluminum pan and allowed to dry at $60^{\circ} \mathrm{C}$. The agar was weighed after drying then was grinded and milled. After preceded all the process the percentage of agar yield was calculated by finding out how much of the Clean Anhydrous Weed (CAW) is equivalent to the powdered agar produced. 


\author{
Weight of powdered agar $\mathrm{x} 100=\%$ of agar yield \\ Weight of CAW
}

Sodium alginate yield $\times 100=\%$ yield of sodium alginate Wet weight of Turbinaria

\section{Sodium alginate}

Extraction of sodium alginate by Green's process: The seaweed samples were collected during high tide session (mostly 4-6 pm) from March-April 2015 Turbinaria decurrens and identified and studied for research studies. After collection and identification, the sample was cleaned by washing with fresh water and was treated with $0.3 \%$ of aqueous $\mathrm{HCl}$ for $30 \mathrm{~min}$. After that the sample was chopped by a pair of scissors and shredded in to small pieces. Furthermore, the shredded sample was treated with $8 \%$ of aqueous $\mathrm{Na}_{2} \mathrm{CO}_{3}(\mathrm{pH} 10-11)$. Then the process was repeated for the second time before solid placement. After that the sample was diluted with water and filtration technique was modified by normal sieve instead of filter aids. The filtered sample was then allowed to heat directly at 500C for 20 minutes on heater. Then the filtrate was mixed with $12 \%$ of aqueous $\mathrm{CaCl}_{2}$ solution and allowed to precipitate. The precipitated sample was separated and the lower liquid layer of solution was drained off and discarded. At last but not least the calcium alginate was bleached with $10 \%$ of aqueous $\mathrm{NaOCl}$ followed by drained and mixed with $5 \%$ of $\mathrm{HCl}$. The produced alginic acid was converted to sodium alginate by adding $5 \%$ of $\mathrm{Na}_{2} \mathrm{Co}_{3}$. A powder form sodium alginate was produced by putting the gel in an oven at $100^{\circ} \mathrm{C}$ for 8 hours. At last the dried sodium alginate was grounded by mortar and pestle and packed.

Hence, the percentage yield of the obtained alginate was calculated as follows:

Sodium alginate yield X $100=\%$ yield of sodium alginate

Wet weight of Turbinaria

\section{Result analysis}

Scientifically one product need to be ensured either physically or chemically that targeted result was obtained. Hence, for sodium alginate referring to European Pharmacopoeia standard chemical analysis of $\mathrm{P} / \mathrm{A}$ identification was done;

a. First $0.2 \mathrm{gm}$ of sample was dissolved in $20 \mathrm{ml}$ of aqua fresh water and $0.5 \mathrm{ml}$ of $\mathrm{Na}_{2} \mathrm{CO}_{3}$ was added to the solution. After shaking and filtered, to $5 \mathrm{ml}$ of the $20 \mathrm{ml}$ filtrate $1 \mathrm{ml}$ of $\mathrm{CaCl}_{2}$ solution was added. As a result, a voluminous gelatinous mass was formed which showed a positive result and indicated sodium alginate was present.

b. To $5 \mathrm{ml}$ of the filtrates obtained in the identification test A, $0.5 \mathrm{ml}$ of a $123 \mathrm{gm} /$ liter solution of $\mathrm{MgSO}_{4}$ was added. After some shake a non-precipitate liquid with very less alginate (non-voluminous gelatinous mass) was formed, which showed a positive result. This indicates the positive result and indicated sodium alginate was present.

\section{Results and discussion}

\section{Agar}

It was obtained from the sea weeds, used as the main sources for the "future Medicinal foods". Earlier it was obtained from the red algae (Rhodophyta) which owns a phycoerythrin pigment for its coloration and besides to that a widely applied hydrocolloid which termed as agar; literally meant as gelatinous substance are highly found in their cellulose [1]. Hence from all Rhodophyta species, Gracilaria and Gelidium are the most dominant industrial seaweeds for agar extraction due to their performance in yielding agar with high quality and quantity [9], comparatively from both species Gracilaria are the best selected species than Gelidium, due to their high cultivation property, increased availability and subsequent competitive price. Having this in consideration this project selected Gracilaria, specifically arcuata species, since they have a diversified distribution in Red Sea and high yield of agar product.

In the extraction procedure samples were collected from Gurgussum and Hirgigo, the sea status should be in low tide session (mostly 6:00 am- 12:00 pm). In which Gurgussum and Hirgigo were about $12 \mathrm{Km}$ and $20 \mathrm{Km}$ respectively from College of Marine Science and Technology (COMSAT). The main reason why low tide preferred was due to their physical morphology, in which they were short with about $3 \mathrm{~mm}$ thickness, with irregularly branched and sometimes unilateral feature but in high tide session, since currents and waves interrupt the collection program it was such unadvisable choice. As a continuation to collection, as long as there weren't any professionals in Botany, mounting of herbarium identification was done by comparing the samples with Atewebrhan studies, internet explorers and some identification key manuals. As a result, the general morphology of Gracilaria arcuata was studied deeply on the way and mostly the species were attached with hard substrates like coral reefs. Hence they had a lot of residues they washed out with enough amount of fresh water in order to extract a pure agar. It was obtained in dryied form or as raw material in order to supply it to other countries which produce agar in large scale. Therefore, this research paper followed up the drying process by using both oven and sun drying during the four trials practiced. In sun drying, simply the sample spread on mats and the sun rays allowed striking on the sample directly. Even though oven drying was the best technique in its safety and duration, but since the vacuum oven dryer of the MBT lab had some disorder the process couldn't be feasible. Irregular temperature increase or decrease and unequal distribution of temperature were some of the problems observed during the drying process. As a result of this, some samples were burned earlier and others were remaining wet. So, in order to avoid such problems sun drying was applied by spreading on mats till they became hard and brittle in order to safe samples from being ruined.

Most of the times the average wet weight of the collected samples were 3 kilograms when measured by weight balance, 
which reduced to about 250 grams after dried since most part of seaweed is water [4]. Having this in mind it was such a wise decision to dry the sample in fields because after drying the weight reduces significantly and this had a great advantage in freeze and thaw process. But there were factors like cleanness of sample and drying method which needed to be included in order to avoid fluctuations during extraction procedures.

Since extraction of agar had been practiced for a long time it showed a lot of modifications, one of the chemical improvements done in that field was, the idea of "pre-extraction treatment" [3]. In which according to the study held in 1990, gel strength of the produced agar could possibly increase by treating samples with water, lime and alkaline solutions. Hence, samples of Gracilaria arcuata were treated first by soaking the dried sample in enough amount of aqua fresh water till they reform their shapes and became bleached. Soaking the dried sample in aqua fresh water was done to remove the salts and other impurities and the drying process repeated for second time by spreading the sample on tables under fan instead of sun drying, in order to avoid dust particles from outdoors. For further bleaching and increasing gel strength 1.3 litter of lime (CaCO3) suspension were prepared and sprinkled on the drying seaweed, since lime has a property of increasing agar gel strength. And obviously the higher the gel strength, the best qualified agar produced.

As a continuation to the pre-extraction treatment, alkaline treatment was followed in order to remove the sulfates present and this treatment was very crucial because sulfates decrease the gel strength of the agar. Compiled in 1990 by Gertrude, there are different alkaline treatments applied depending upon the climatic conditions. By comparing all the methods and factors, alkaline treatment protocol of Chile was selected since it had similar climatic condition as Massawa (especially from February till April session). In which $10 \%$ of aqueous sodium hydroxide solution was added for the bleaching purpose and water bathed at $95^{\circ} \mathrm{C}$ for 90 minutes. Even though the gel strength couldn't be measured in this research paper but according to Kim (1970), gel strength of $910 \mathrm{~g} / \mathrm{cm}^{2}$ (1.5\% conc.) could be obtained by following the same alkali treatment. Hence the applied $\mathrm{NaOH}$ was in its concentrated form they left residues over the samples, therefore in order to free the samples from alkaline they were washed over and over again. As a result of this the reddish colored Gracilaria turned to pale yellowish almost white color and this indicated that the pre extraction treatment process has a significant effect on color change. Hence, the white color obtained was strong evidence that the alkali treatment was effective. After all this the sample was dried by following same as previous techniques and the average weight of the treated sample was reduced again to $125 \mathrm{gm}$. This further reduction of the sample weight could be either due to bio mass loss during heating on water bath at $95^{\circ} \mathrm{C}$ for 90 minutes or due to loss of some residue during extensive washing of the samples with fresh water to remove $\mathrm{NaOH}$ after alkali treatment.

Extraction process was followed after pre extraction treatment and was started by partitioning the dried sample to beakers, where enough amount of acetic acid solution was passed through, till it reached a pH range of 5.6-6.0. After soaking the treated Gracilaria for 30 minutes, the dried sample retained its size and became totally bleached so that they seemed as the same as they were fresh. Since the concentration of the acetic acid; which had the ability to further remove the phycoerythrin pigment by breaking the cellulose was $99.5 \%$ which was concentrated, they obliged to be diluted in order to prevent further lysis of the cells by adding $1000 \mathrm{ml}$ of aqua fresh water. In addition to that, direct heat was applied even though water bath was the written procedure to follow up, in order to modify the yellowish color of the product and to a whitish gel. In the first three trials the color of the agar was yellow or pale yellow and this was due to usage of water bath so in order to improve the color of the agar the process was modified by using direct boiling or heating instead of water bath. As a result of this the obtained agar was white and transparent and this modification played a great role in the success of the extraction of agar.

Not only this was enough, for further extracting of the remained agar the heated sample passed to a blender. Since the samples were bleached physically the blended result looked like a "banana juice" with weak gel. After blending the gooey extract returned to a pan, the blender washed with hot water that boiled during the process and the washing was mixed with the rest of the extract. According to further heating for three hours increases the gel strength of the obtained agar [8]. So, as a continuation in order to increase the gel strength the same procedure as Gertrude was followed and very viscous agar was produced. But since there were residues in solutions of the blended samples, filtration technique was applied. According to procedure, filter aids were applied but since they were not economically feasible normal filter was applied [8]. After filtration, the extracted agar was allowed to gel at room temperature and after one hour an incredible gel was formed. [8] According to the content of the obtained gel is mostly water and this water was instructed to be removed by repeatedly freeze and thaw process and the same principle was used. In this case the freeze and thaw process was undertaken for three days and enough amount of water was removed. At last but not least an agar with a gel, which retained the shape of the container, were obtained. And for further preservation they changed into powder form; in which the thawed agar was dried in an oven for 6 hours at $60^{\circ} \mathrm{C}$ and mathematically, as shown in below calculation $20.4 \%$ of agar was produced.

$\frac{\text { Weight of powdered agar x } 100}{\text { Weight of CAW }}=\%$ of agar yield

$$
\frac{25.5 \text {-gram }}{125 \text { gram }} \times 100=20.4 \% \text { of agar yielded }
$$

\section{Sodium Alginate}

Alginates as they are part of the hydrocolloids they play a great role in diversifying humans' interest. Scientists had 
a specific survey on finding out which genius and species of Phaeophyta could yield high quality alginates, and as a result of that time foundation by Younis. et al. (2000), Turbinaria ornata is the best candidate species in its quality and yield, even though Sargassum species are the diversified ones. In Eritrea as many scientists including Atewebrhan studied in 2005, the distribution of Turbinaria ornata on Eritrean Red Sea is mainly on islands like Dur-gam; which was far away from Massawa College of Marine Science and Technology (COMSAT). Hence, as an alternative Turbinaria decurrens which could be found on the coastal reefs of Red Sea near Twalet were selected in completing this senior research project.

Sodium alginate was extracted using preferable method which was the green process from all the five mostly used protocols, since Green process contained the materials and chemicals found in MBT lab of COMSAT. Hence, seaweed was the main raw material for alginate extraction, they were then collected from coastal reefs of Twalet region by cutting the thallus with hand near the rhizoid during high tide session (mostly around 4:00 pm to 6:00 pm). The exact reason why high tide session was chosen over low tide was that, during low tide most of the coral reefs and sea urchins in that area were exposed out and their tips were sharp; which makes the collection process difficult and inconvenient, by leading to sever health effects. Having those fears in mind, collection process was succeeded through snorkeling technique by the help of goggles and flippers borrowed from department of Marine Biology and Fisheries (MBF). As long as the specific species name of the collected genius had to be found out, herbarium identification was practiced by referring $[2,12]$. As a result, they turned out to be called as Turbinaria decurren.

Unlike the other seas in the world the salinity of red sea is very high since it doesn't have an interaction and flow with other water bodies to dilute the percentage of salinity [3]. Hence the collected sample was washed extensively with enough amount of fresh water till the salts, sand, shells and unwanted substances were removed. Since the initial weight of the sample required in order finding out the percentage yield of the product obtained, they measured by using beam balance and resulted as $500 \mathrm{gm}$. Like all phycocolloids, alginates also found out to be inside the cellulose part. Therefore, in order to break the outer part (cellulose), the treated sample was leached with $0.3 \%$ of aqueous $\mathrm{HCl}$ acid for 30 minutes and shredded with pair of scissors to increase the surface area. Since the aim of extraction was to form a gel, the shredded sample was treated with $8 \%$ of alkali solution $\left(\mathrm{Na}_{2} \mathrm{CO}_{3}\right)$ at $\mathrm{pH} 10$ for 1 hour; as a result, very thick slurry of reddish color was formed. In which the slurry had contained part of the seaweed that doesn't dissolve. This showed that the cellulose (alginate) found in brown algae can easily be dissociated unlike the Rhodophyta species. Hence, the process repeated for the second time before solid placement in order to increase the gel formation and diluted with water to reduce the concentration of the chemicals applied and volume of the insoluble residues.

As a continuation to the extraction process, direct heat was applied to the treated Turbinaria species by putting them in an aluminum pan (for more safety) at $50^{\circ} \mathrm{C}$ for 30 minutes. After heating since the solution was too thick (viscous) to be filtered $100 \mathrm{ml}$ of aqua fresh water was added and forced through a normal sieve. However, since the pieces of the residue were very fine they quickly passed through the normal sieve prepared. Therefore, before filtration started the sieve was covered with chiffon cloth to reduce the size of the pores instead of filter aids which are very expensive.

Generally, all in all three trials of sodium alginate had been practiced in this research, in which the procedure of the first two trials differed in their ways of recovering. That was in first trial unlike Green process which mixed the filtrates with $12 \%$ of aqueous $\mathrm{CaCl}_{2}$, they recovered by addition of acid $(\mathrm{HCl})$ and the third trial was the same as the second trial which was successful.

\section{First trial ( $\mathrm{HCl}$ acid recovering technique)}

When the viscous gel filtered, again hydrochloric acid of 5\% was added to the solution in the aluminum pan, as a result white bubbles were lifted on the surface where they could continuously have scrapped away and jelly-like mass of alginic acid were produced. To the knowledge of our understanding the suspension formed might be due the specific binding activity of the $\mathrm{HCl}$ acid chemical used with the bond of the alginates. Since the end product should be in powder form the water content present in the slurry solution of alginic acid was removed by precipitation technique. When the gel of alginic acid settled down the water floated in the upper part. So to separate the precipitates filtration process was followed and a reddish colored liquid was drained off. After this has been done alcohol (ethanol) was added to the alginic acid for bleaching purpose, since alcohols in general have chemically high affinity to interact with the cell membrane of organisms. Hence the main aim of the study was to extract sodium alginate, $8 \%$ of $\mathrm{Na}_{2} \mathrm{CO}_{3}$ was added and alginic acid converted into sodium alginate. As a result, reddish colored product similar to that at first was obtained. In which comparing with the physical property of the industrially produced alginates, it was not exactly sodium alginate.

\section{Second trial (Calcium salt recovering technique)}

The procedure was the same till the filtration process, in which the second trial differed since $12 \%$ of aqueous solution was added and stirred for 15 minutes. As a result of the chemical applied white condensed suspension reaction was formed. That was mostly because, chemically $\mathrm{CaCl}_{2}$ had the property of changing colour; as a result, the previous reddish like color turned into pale yellowish white color, which was one of the physical properties of sodium alginate. Hence the jelly product was on upper part; a reddish colored liquid part was remained in the lower part and discarded off. But, for better bleaching process $10 \%$ of aqueous $\mathrm{NaOCl}$ solution was added and stirred well till the chemicals distributed throughout the calcium alginate. After that the inserted $\mathrm{NaOCl}$ drained off and in order to remove the calcium salts which had been applied for the sake of precipitation 
$5 \% \mathrm{HCl}$ acid was added. As a continuation the treated sample was washed with enough amount of aqua fresh water to remove the excess chemicals applied. Then alginic acid was treated by $\mathrm{Na}_{2} \mathrm{CO}_{3}$ in order to produce sodium alginate. After an overnight precipitation, next day as shown in figure $10 \mathrm{~A}$ the precipitated sodium alginate was partitioned into three layers according to their gel strength. In which the upper layer had very weak gel comparing with the two and the middle alginate was moderate, but the bottom part had the greater gel from all. This represented that there was some suspended water left during filtration process. At last, in order to convert the product obtained into powder form, sodium alginate entered into an oven at $100^{\circ} \mathrm{C}$ and after 6 hours' time they completely dried and milled with mortar and pestle.

Mathematically, as shown in below calculation $25.6 \%$ of sodium alginate was produced.

Weight of powdered sodium alginate $\mathrm{x} 100=\%$ of sodium alginate yield

Weight of CAW of Turbinaria

128 gram $\times 100=25.6 \%$ of sodium alginate yielded

Over all, scientifically when one product produced it needs to be ensured either physically or chemically that targeted result was obtained. Hence, for sodium alginate referring to European Pharmacopoeia standard chemical analysis of P/A identification was applied. In that case the powdered sodium alginate was testified by using two methods. In the first method voluminous gelatinous mass was formed and this was due to the addition of $\mathrm{CaCl}_{2} \cdot \mathrm{CaCl}_{2}$ binds with sodium alginate which causes precipitation reaction and this was due to the chemical property of $\mathrm{CaCl}_{2}$. But in second case after the addition of $\mathrm{MgSO}_{4}$ no voluminous gelatinous mass was formed because MgSO4 does not have precipitation reaction with sodium alginate. As this analysis was $\mathrm{P} / \mathrm{A}$ test in the first case the precipitation reaction of $\mathrm{CaCl}_{2}$ indicates the presence of sodium alginate. But in the second case since no precipitate reaction was formed, this analysis confirmed the presence of sodium alginate because according to European Pharmacopoeia standard chemical analysis reaction of sodium alginate and $\mathrm{MgSO}_{4}$ doesn't form any precipitation reaction. So that it was confirmed that the extracted product was sodium alginate.

\section{Conclusion}

Agar and alginate are extracted from marine red algae (Gracilaria arcuata) and brown algae (Turbinaria decurren) which has a great role in binding pharma and agro-based industry. Hence, the importance of sea weeds is of great use to human beings.

\section{Recommendation}

- proper exploitation is required for extraction of sea weed as byproducts

- cost benefit analysis in terms of high percentage of yield

\section{References}

1. Armisen R, Galatas F. production and utilization of products from commercial seaweeds, FAO Fish.Tech.pap.288, 189 pp. 1987. Production, properties and uses of agar. pp.1-57. In: McHugh, D.J. (ed.)

2. Ateweberhan M, Prud'homme van Reine W. A taxonomic survey of seaweeds from Eritrea. BLUMEA. 2005;50(1):65-111.

3. Barnes K, Hughes RN. An introduction to marine ecology. 2nd edition. Blackwell science. Britain. ISBN 0-632-02049-0. 1988.

4. Barsanti L, Gualtieri P. Algae: anatomy, biochemistry and biotechnology. CRC press. New York. ISBN. 2006. 13:978-0-84931467-4.

5. Chandrkrachang S, Chinadit U. Seaweed production and processing: a new approach. INFOFISH Inter. 1988;4:22-25.

6. Duckworth M, Hong KC, Yaphe W. The agar polysaccharides of Gracilaria species. Carbohydrate Research. 1971;18(1):1-9.

7. Food and Agriculture Organization (FAO). (2006) State of World Aquaculture: 2006 FAO Fisheries Technical Paper 500. Inland Water Resources and Aquaculture Service Fishery Resources Division Fisheries Department, Rome 2006.

8. Santos GA. A manual for the processing of Agar from Gracilaria. ASEAN/SF/90/Manual No.5.1990.

9. Hernandez-Carmona G, Freile-Pelegrin Y, Hernandez Garibay E. Conventional and alternative technologies for the extraction of algal polysaccharides. Journal of Applied Phycology. 2013;23:321335.

10. Khanafari A, Sepahei AA. Alginate biopolymer production by Azotobacter chroococcum from whey degradation. International Journal of Environmental Science \& Technology. 2007;4(4):427-432.

11. Lee B. Seaweed potential as marine vegetable and other opportunities. Can print, Australia. ISBN 174151 598. 2008.

12.Lieske E, Myers FR. Coral reef Guide Red Sea. Printing express. Hong Kong. ISBN-13: 978000715986 4. 2004.

13. Matsukawa R, Dubinsky Z, Kishimoto E, Masaki K, Masuda Y, Takeuchi T, et al. A comparison of screening methods for antioxidant activity in seaweeds. Journal of Applied Phycology. 1997;9:29-35.

14. Mc Williams M. FOODS: experimental perspectives. 6th edition. Hamilton press. USA. ISBN. -13: 978-0-13-156853-2. 2008.

15. McDonell MW, Simon MN, Studier FW. Analysis of restriction fragments of T7 DNA and determination of molecular weights by electrophoresis in neutral and alkaline gels. J Mol Biol. 1977;110(1):119-146.

16. Nisizawa K. Seaweeds Kaiso Bountiful Harvest from the Sea. In Seaweed Resources of the World (Critchley AT, Ohno M, Largo DB editors). Japan International Cooperation Agency, Yokosuka. 2006.

17. Peggy V, Raymond K, Abdellah A, Regis B, Legrand J. A new process for extracting alginates from Laminaria digitata: Reactive extrusion. Food and Bioprocess Technology. 2008;3(1):297-300.

18. Khan SI, Satam SB. Seaweed mariculture: Scope and potential in India. Am J Biochem Biotechnol. 2003;1:56-78.

19. Southern E. [9] Gel electrophoresis of restriction fragments. Methods in Enzymology. 1979;68:152-176.

20.Vauchel P, Kaas R, Arhaliass A, Baron R, Legrand J. A new process for extracting alginates from Laminaria digitata: Reactive Extrusion. Springer. 2008;3(1):297-300.

21. Gates KW. Marine products for healthcare: Functional and Bioactive Nutraceutical Compounds from the Ocean, Vazhiyil Venugopal. Journal of Aquatic Food Product Technology. 2010;19(1):48-54. 